\title{
CLASSIFICAÇÃO DO MEL EM FLORAL OU MEL DE MELATO ${ }^{1}$
}

\author{
G. CAMPOS ${ }^{2, *}$, R. C. DELLA-MODESTA ${ }^{3}$, T. J. P. SILVA ${ }^{4}$, K. E. BAPTISTA ${ }^{2}$,
}

M. F. GOMIDES ${ }^{2}$, R. L. GODOY

\begin{abstract}
RESUMO
O mel é um produto natural de abelhas fabricado a partir do néctar das flores (mel floral) e de secreções de partes vivas das plantas, ou de excreções de insetos sugadores de partes vivas das plantas (mel de melato). O melato é uma fonte alternativa de alimento bastante utilizada pela abelha. Em Santa Catarina, o mel de melato é muito conhecido, sendo produzido nos meses de janeiro a abril, em ciclos bianuais. Foram colhidas aleatoriamente 25 amostras de mel, no periodo de janeiro/93 a junho/96, de diferentes apiários e datas de colheita. Foram determinados os teores de umidade, $\mathrm{pH}$, cinzas e açúcares redutores. Os resultados foram utilizados na aplicação da equação de Kirkwood para classificar o mel como floral ou de melato. As amostras também foram analisadas quanto à rotação óptica para classificação de acordo com White. Das 25 amostras analisadas, 11 foram classificadas como mel de melato de acordo com Kirkwood e White. O estudo estatístico, feito pela aplicação do teste exato de Fisher, indicou que, apesar da divergência em 4 amostras, estes métodos são equivalentes e ambos podem ser usados.
\end{abstract}

Palavras-chave: mel de melato; composição fisico-quimica; métodos analíticos.

\section{SUMMARY}

CLASSIFICATION OF HONEY AS FLORAL OR HONEYDEW HONEY. Honey is a natural product made by bees using flower's nectar (floral honey) or excretion of alive parts of plants or either excretion produced by sucking insects from alive parts of plants (honeydew honey). Honeydew is an alternative food source used by bees. In Santa Catarina state, honeydew honey is produced from january to april, each two years. In order to identify the presence of honeydew honey, 25 samples were sampled randomly from january/93 to june/96. They were colected from different apiaries in different periods of time and tested for moisture, $\mathrm{pH}$, ash, reducing sugars, and the results were used in the application of Kirkwood equation to classify honey as floral or honeydew. They were analised for polarimetry, to classify them according to Kirkwood and White. The statistical study was done by the application of exact test of Fisher and, in spite of some divergences found to classify 4 samples, the statistical study showed correlation between Kirkwood and White methods, indicating that both can be used.

Keywords: honey; honeydew in honey; physical and chemical composition.

\section{1 - INTRODUÇÃO}

O mel, por definição, é um produto natural de abelhas obtido a partir do néctar das flores (mel floral), de secreções de partes vivas das plantas ou de excreções de insetos sugadores de partes vivas das plantas (mel de melato).

Melato é um vocábulo que, em biologia, refere-se às excreções, em forma de líquidos açucarados, de um grande número de espécies de homópteros que vivem como parasitas sugadores da seiva elaborada do floema das plantas. Estes líquidos açucarados que são procurados e colhidos pelas abelhas como se fossem néctar, passam pelos mesmos processos enzimáticos. O produto final, entretanto, é diferente nas suas propriedades fisico-químicas e constitui o mel de melato [3].

Em Santa Catarina ocorre o mel de melato do caule da bracatinga (Leguminosae Mimosoideae Mimoso sp), produzido de dois em dois anos, época que corresponde ao ciclo da cochonilha. Além da bracatinga há também produção de melato a partir do ingá. Como regiões pro-

1. Recebido para publicação em 07/01/1999. Aceito para publicação em 15/05/2002.

2. Fundação Ezequiel Dias - Unidade Gerência da Qaulidade - IOM Rua Conde Pereira Carneiro,80 - B. Gameleira. CEP 30510-010. Belo Horizonte-MG

3. Embrapa - Agroidústria dos Alimentos - Rio de Janeiro-RJ.

4. Universidade Federal Fluminense - UFF.

* A quem a correspondência deve ser enviada. dutoras de mel de melato podem ser citados Planalto Sul, Município de Bom Retiro, Urubici, Vale do Canoas, Lages, Painel, Pocama do Sul, S. Joaquim, Santa Terezinha e Alto Vale do Itajaí. Na década de 80, o entreposto de mel e cera de abelhas "Colonial de Itajai"- SC iniciou a exportação do produto para a Alemanha, a fim de ser utilizado na elaboração de fármacos. No final de 2000, o entreposto de mel e cera de abelha "Miramel" Ltda., Içara - SC, conseguiu exportar para a Alemanha este mesmo mel por 1400 dólares a tonelada, versus 1100 dólares a tonelada do mel floral $[8,12]$. A Figura 1 mostra a Bracatinga, em Bom Retiro, Santa Catarina.

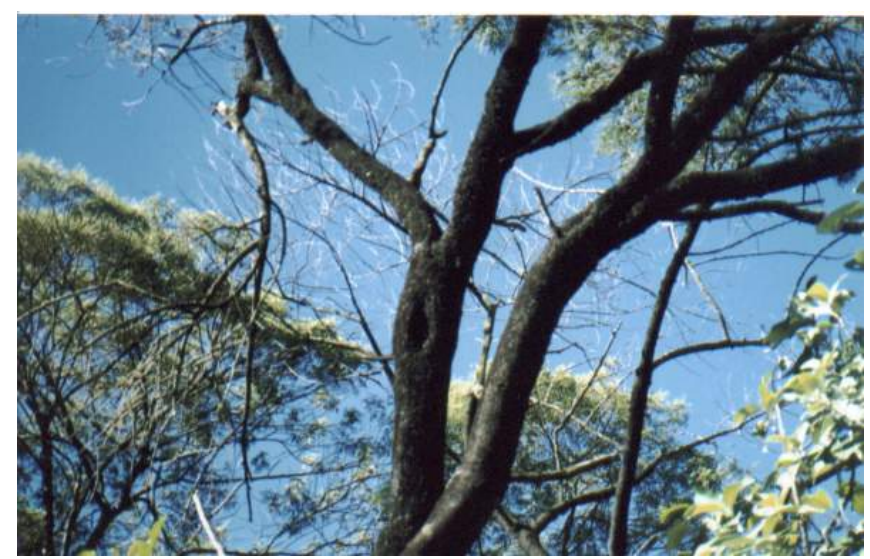

FIGURA 1. Vegetação de Bracatinga em Bom Retiro, S.C. 
O mel é uma matriz muito complexa, havendo, durante a sua elaboração, interferência de variáveis não controladas pelo homem, como clima, floração, presença de insetos sugadores e outros fatores. As abelhas, por sua vez, vão utilizar os recursos disponiveis como fonte de açúcar para elaborá-lo. Portanto, o mais comum é a ocorrência de mel floral misturado com mel de melato.

O mel de melato difere do mel floral em vários aspectos: por exemplo, o mel de melato possui menor teor de glicose, razão pela qual usualmente não cristaliza; este tipo de mel apresenta também menor teor de frutose, maior teor de oligossacarídeos e de cinzas, maior $\mathrm{pH}$ e maior teor de nitrogênio [7, 13].

A fim de determinar a ocorrência de melato no mel, KIRKWOOD [9, 10] analisou várias amostras de mel floral e de mel de melato e, após verificar os parâmetros que mais variaram, estabeleceu uma equação baseada em estudos matemáticos para ser aplicada utilizando os resultados de $\mathrm{pH}$, cinzas na matéria seca e açúcares redutores na matéria seca obtendo uma função discriminativa $\mathrm{X}$ e estabelecendo um valor limite para diferenciação entre estes dois tipos de mel. Foi adotado o valor limite de 73,1, abaixo do qual o mel é classificado como mel de melato.

Considerando empírico o conceito de que amostras de mel são levorrotatórias e melato dextrorrotatórias e que não existe embasamento real nesta divisão, exceto no fato de que melezitose e erlose, presentes no mel de melato, são fortemente dextrorrotatórias, WHITE [14] determinou a rotação ótica de 468 amostras de mel e converteu os valores encontrados para a "International Sugar Scale". O valor médio encontrado para as 454 amostras levorrotatórias foi de $-14,70^{\circ} \mathrm{S}$, com desvio padrão de $4,37^{\circ} \mathrm{S}$ e $\mathrm{CV}=29,77 \%$. Este autor propôs um limite de $-2^{\circ} \mathrm{S}$, ao invés de $0^{\circ} \mathrm{S}$, para indicar mel com apreciável teor de melato. O mel de melato, sendo uma ocorrência natural, precisa ser caracterizado e identificado para que seja possivel haver um controle sobre a qualidade do produto. É preciso estar atento com o mercado consumidor para que haja uma boa aceitação, principalmente pelo mercado externo que é mais exigente, inclusive o do Mercosul. É de fundamental importância que o produto tenha bem definido seus padrões de identidade e qualidade. Por isso, o mel tem que ser analisado a fim de se estabelecer a sua origem: floral ou melato. A legislação atual exige que no rótulo esteja discriminado melato ou mel de melato nos méis de melato ou nas misturas de mel floral e mel de melato [5].

\section{2 - MATERIAL E MÉTODOS}

\section{1 - Material}

Foram analisadas 25 amostras de mel colhidas aleatoriamente entre o periodo de 02/01/93 a 30/06/96 de diferentes apiários e/ou em datas diferentes (Quadro 1). As amostras provenientes de Santa Catarina (4, 5, 22 e 23) são mel de melato do caule da bracatinga [6].
QUADRO 1. Procedência das amostras de mel

\begin{tabular}{|c|c|c|}
\hline $\begin{array}{c}\mathrm{N}^{\mathrm{o}} \mathrm{da} \\
\text { amostra }\end{array}$ & Procedência & Colheita \\
\hline 1 & Caeté - MG & $10 / 06 / 95$ \\
\hline 2 & B. Cocais $-\mathrm{MG}$ & maio/95 \\
\hline 3 & B. Cocais $-\mathrm{MG}$ & maio/95 \\
\hline 4 & Bom Retiro -SC & maio/94 \\
\hline 5 & S. Catarina & $1992 / 93$ \\
\hline 6 & B. Cocais $-\mathrm{MG}$ & $09 / 09 / 95$ \\
\hline 7 & B. Cocais -MG & $09 / 09 / 95$ \\
\hline 8 & Passa Quatro-MG & $23 / 09 / 95$ \\
\hline 9 & Ipatinga $-\mathrm{MG}$ & $23 / 05 / 95$ \\
\hline 10 & Paraopeba-MG & 1993 \\
\hline 11 & Itatiaiussu - MG & nov/95 \\
\hline 12 & Diamantina $-\mathrm{MG}$ & nov/95 \\
\hline 13 & Santa Luzia - MG & $\operatorname{dez} / 95$ \\
\hline 14 & Barra Longa - MG & $\mathrm{jan} / 96$ \\
\hline 15 & Mariana - MG & jan/96 \\
\hline 16 & B. de Cocais - MG & $\mathrm{fev} / 96$ \\
\hline 17 & B. de Cocais - MG & $14 / 2 / 96$ \\
\hline 18 & B. de Cocais - MG & $12 / 1 / 96$ \\
\hline 19 & B. de Cocais - MG & $15 / 2 / 96$ \\
\hline 20 & B. de Cocais - MG & $13 / 4 / 96$ \\
\hline 21 & B. de Cocais - MG & $30 / 4 / 96$ \\
\hline 22 & Sta. Catarina & $09 / 5 / 96$ \\
\hline 23 & Sta. Catarina & Julho/96 \\
\hline 24 & B. de Cocais - MG & $28 / 09 / 96$ \\
\hline 25 & B. de Cocais - MG & set/96 \\
\hline
\end{tabular}

A Figura 2 mostra uma das amostras provenientes de Santa Catarina. As demais amostras na sua maioria não apresentavam rótulos, sendo obtidas diretamente do produtor.

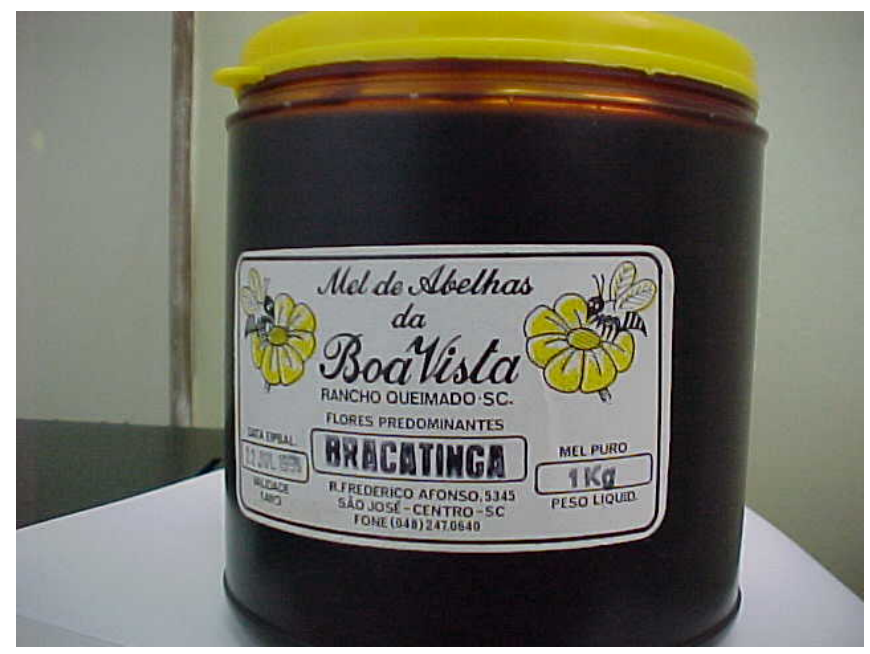

FIGURA 2. Amostra de mel de melato da bracatinga.

\section{2 - Métodos}

\subsection{1 - Preparo da amostra}

Mel líquido: a amostra, livre de granulação, foi bem homogeneizada com o bastão de vidro. Quando granulado, colocou-se o mel em recipiente fechado em banho- 
maria sem submergir e aqueceu-se por $30 \mathrm{~min}$ a $60^{\circ} \mathrm{C}$. Posteriormente, aqueceu-se a $65^{\circ} \mathrm{C}$ ate liquefazer, agitando-se ocasionalmente. Misturou-se, resfriou-se e pesaram-se porções das amostras para as determinações.

\subsection{2 - Determinação da umidade}

Foi feita pela determinação do índice de refração a $20^{\circ} \mathrm{C}$ e pela Tabela de Chataway, de acordo com as NORMAS ANALÍTICAS DO INSTITUTO ADOLFO LUTZ [11].

\subsection{3 - Cinzas}

Foi realizada a incineração da amostra em bico de Bunsen e mufla a $550^{\circ} \mathrm{C}$, de acordo com as NORMAS ANALÍTICAS DO INSTITUTO ADOLFO LUTZ [11].

\subsection{4 - Determinação do $\mathrm{pH}$}

A determinação de $\mathrm{pH}$ foi realizada com um $\mathrm{pHmetro}$ digital marca Procyon, pH10, de acordo com as normas técnicas do Lanara [4].

\subsection{5 - Açúcares Redutores (AR) por espectrofotometria de absorção molecular na re- gião do visivel}

Foi preparada uma solução de mel a 1\% e clarificada com creme de alumina. Desta solução foi preparada a solução de trabalho a $0,05 \%$, da qual pipetou-se $1 \mathrm{~mL}$ para a reação com o ácido 3,5- dinitrossalicílico. Foi preparada a curva padrão de glicose e a leitura foi feita a 540nm [1].

\subsection{6 - Aplicação da equação de Kirkwood}

Os resultados de $\mathrm{pH}\left(\mathrm{x}_{1}\right)$, cinzas $\left(\mathrm{x}_{2}\right)$ e AR $\left(\mathrm{x}_{3}\right)$, sendo os dois últimos calculados na matéria seca, foram utilizados na equação de Kirkwood, para obtenção da função discriminativa:

$X=-8,3 x_{1}-12,3 x_{2}+1,4 x_{3}$

Sendo X menor que 73,1 o mel foi considerado como de melato.

\subsection{7 - Polarimetria}

Equipamento: polarímetro digital Perkin Elmer 341, com lâmpada de $\mathrm{Na}$ a $589,3 \mathrm{~nm}$ e tubo de $100 \mathrm{~mm}$ de comprimento, acoplado a banho de água circulante a $20^{\circ} \mathrm{C}$.

Reagentes:

- creme de alumina

Procedimento:

Pesaram-se $26 \mathrm{~g}$ de mel e transferiu-se para balão volumétrico de $100 \mathrm{~mL}$ com água destilada. Adicionouse cerca de $1 \mathrm{~g}$ de creme de alumina, completou-se o volume e filtrou-se. Para completar a mutarrotação a amostra ficou em repouso 24 horas antes da leitura que foi feita a $20^{\circ} \mathrm{C}[2]$.

\section{- Cálculo em ${ }^{\circ} \mathrm{S}$}

Os valores de rotação angular foram convertidos para a International Sugar Scale ( ISS ), aplicando-se a fórmula seguinte:

${ }^{\circ} \mathrm{S}=\underline{\text { Leitura } \times 2 \times 0,26}$

0,3462

$1^{\circ} \mathrm{S}=0,3462$ graus angulares. A multiplicação por 2 deve ser feita somente quando o tubo utilizado for de $1 \mathrm{dm}$, pois os valores em ISS requerem tubo de $2 \mathrm{dm}[14]$.

\section{3 - RESULTADOS E DISCUSSÃO}

TABELA 1. Valores médios (repetições)* de umidade, cinzas, $\mathrm{pH}$ e açúcares redutores em amostras de mel e classificação conforme KIRKWOOD [9, 10]

\begin{tabular}{|c|c|c|c|c|c|c|}
\hline Amostra & $\begin{array}{l}\text { Umidade } \\
(\%)\end{array}$ & $\begin{array}{c}\text { Cinzas } \\
(\%)\end{array}$ & $\mathrm{pH}$ & $\begin{array}{l}\text { Açúcares } \\
\text { redutores }\end{array}$ & $\begin{array}{c}\text { X } \\
\text { (Equação de } \\
\text { Kirkwood) } \\
\end{array}$ & Classificação \\
\hline 1 & 19,6 & 0,39 & 4,1 & $71,40 \pm 0,18$ & 84,53 & mel floral \\
\hline 2 & 18,8 & 0,31 & 4,2 & $71,48 \pm 1,10$ & 84,01 & mel floral \\
\hline 3 & 16,8 & 0,21 & 4,0 & $70,44 \pm 1,73$ & 82,2 & mel floral \\
\hline 4 & 16,0 & 0,74 & 4,9 & $67,20 \pm 0,48$ & $60,50^{* *}$ & mel de melato \\
\hline 5 & 16,6 & 0,66 & 4,7 & $62,17 \pm 0,95$ & $56,51^{* *}$ & mel de melato \\
\hline 6 & 15,8 & 0,10 & 4,1 & $58,01 \pm 0,49$ & $61,07^{* *}$ & mel de melato \\
\hline 7 & 18,2 & 0,11 & 3,9 & $58,09 \pm 0,29$ & $63,03 * *$ & mel de melato \\
\hline 8 & 15,2 & 0,11 & 4,0 & $59,88 \pm 1,61$ & $70,77^{* *}$ & mel de melato \\
\hline 9 & 19,0 & 0,60 & 4,4 & $71,11 \pm 3,56$ & 77,30 & mel floral \\
\hline 10 & 17,0 & 0,34 & 4,6 & $60,72 \pm 2,83$ & $59,20 * *$ & mel de melato \\
\hline 11 & 18,2 & 0,09 & 4,5 & $65,44 \pm 1,83$ & 73,30 & mel floral \\
\hline 12 & 17,8 & 0,07 & 4,7 & $64,72 \pm 0,43$ & $70,17 * *$ & mel de melato \\
\hline 13 & 19,4 & 0,06 & 4,3 & $61,64 \pm 0,76$ & $70,47^{* *}$ & mel de melato \\
\hline 14 & 19,0 & 0,06 & 4,2 & $65,70 \pm 0,22$ & 77,73 & mel floral \\
\hline 15 & 16,6 & 0,02 & 4,0 & $61,93 \pm 0,03$ & $70,47^{* *}$ & mel de melato \\
\hline 16 & 20,8 & 0,29 & 4,2 & $77,11 \pm 0,44$ & 97,05 & mel floral \\
\hline 17 & 19,4 & 0,21 & 3,8 & $68,97 \pm 0,60$ & 85,06 & mel floral \\
\hline 18 & 18,6 & 0,09 & 4,1 & $70,47 \pm 0,50$ & 85,81 & mel floral \\
\hline 19 & 20,3 & 0,12 & 3,9 & $71,48 \pm 0,33$ & 91,34 & mel floral \\
\hline 20 & 20,8 & 0,06 & 3,9 & $70,51 \pm 0,55$ & 91,28 & mel floral \\
\hline 21 & 17,4 & 0,10 & 4,1 & $73,67 \pm 0,49$ & 83,47 & mel floral \\
\hline 22 & 17,4 & 6,47 & 5,1 & $55,73 \pm 0,98$ & $-43,58 * *$ & mel de melato \\
\hline 23 & 16,1 & 0,28 & 5,3 & $58,22 \pm 0,18$ & $49,16^{* *}$ & mel de melato \\
\hline 24 & 17,7 & 0,11 & 3,8 & $66,06 \pm 0,41$ & 79,32 & mel floral \\
\hline 25 & 16,7 & 0,13 & 4,0 & $66,92 \pm 0,77$ & 77,35 & mel floral \\
\hline
\end{tabular}

** $\mathrm{X}<73,1=$ mel de melato* 2 repetições para umidade, resíduo mineral fixo e $\mathrm{pH}$ 3 repetições para Açúcares Redutores

A Tabela 1 mostra os teores de umidade, cinzas, $\mathrm{pH}$, açúcares redutores e os resultados da aplicação da equação de Kirkwood, considerando como mel de melato a amostra que apresentou X menor que 73,1 .

Na Tabela 2 estão os resultados da polarimetria. De acordo com WHITE [14] as amostras 4, 5, 6, 7, 8, 11, 13, $15,22,23$ e 24 contêm quantidade razoável de mel de melato por apresentarem desvio maior que $-2^{\circ} \mathrm{S}$. As amostras 4, 5, 22, 23, mel de melato da bracatinga, são dextrorrotatórias estando de acordo com a literatura [7].

Na Tabela 3, as amostras foram classificadas em mel floral ou de melato, de acordo com KIRKWOOD et al. e WHITE $[9,10,14]$.

Pode ser observado que 14 amostras foram classificadas como florais e 11 como mel de melato, tanto pela equação de Kirkwood, quanto pela polarimetria, haven- 
do divergência nas amostras 10,11,12 e 24. Dentre estes, alguns resultados da equação de Kirkwood estão muito próximos do limite estabelecido pelo autor, de 73,1.

TABELA 2. Valores da Rotação óptica $\left({ }^{0} \mathrm{~S}\right)$ de amostras de mel (duplicata)

\begin{tabular}{|c|c|c|c|}
\hline Amostra & \multicolumn{2}{|c|}{$\begin{array}{c}{ }^{\circ} \mathrm{SSS} \text { (International sugar scale) } \\
{ }_{\mathrm{o}}\end{array}$} & Classificação conforme White \\
\hline 1 & $-4,186$ & $-4,270$ & mel floral \\
\hline 2 & $-4,592$ & $-4,300$ & mel floral \\
\hline 3 & $-2,276$ & $-2,180$ & mel floral \\
\hline 4 & $+3,217 *$ & $+3,530 *$ & mel de melato \\
\hline 5 & $+1,564 *$ & $+1,730 *$ & mel de melato \\
\hline 6 & $-0,973 *$ & $+0,580 *$ & mel de melato \\
\hline 7 & $-2,010^{*}$ & $-2,290$ & mel de melato \\
\hline 8 & $-1,540 *$ & $0,000 *$ & mel de melato \\
\hline 9 & $-4,374$ & $-4,840$ & mel floral \\
\hline 10 & $-2,537$ & $-3,530$ & mel floral \\
\hline 11 & $-1,703 *$ & $-1,430 *$ & mel de melato \\
\hline 12 & $-3,154$ & $-2,630$ & mel floral \\
\hline 13 & $-1,765^{*}$ & $-1,430 *$ & mel de melato \\
\hline 14 & $-2,930$ & $-2,250$ & mel floral \\
\hline 15 & $-1,006^{*}$ & $-0,980 *$ & mel de melato \\
\hline 16 & $-2,968$ & $-3,230$ & mel floral \\
\hline 17 & $-3,558$ & $-3,760$ & mel floral \\
\hline 18 & $-3,180$ & $-4,200$ & mel floral \\
\hline 19 & $-3,274$ & $-3,600$ & mel floral \\
\hline 20 & $-3,470$ & $-5,180$ & mel floral \\
\hline 21 & $-4,392$ & $-5,330$ & mel floral \\
\hline 22 & $+4,474^{*}$ & $+5,480 *$ & mel de melato \\
\hline 23 & $+3,560$ & $+2,670$ & mel de melato \\
\hline 24 & $-1,200^{*}$ & $-1,500 *$ & mel de melato \\
\hline 25 & $-2,400$ & $-2,400$ & mel floral \\
\hline
\end{tabular}

*Mel de melato conforme WHITE [15]

TABELA 3. Classificação do mel em floral e mel de melato de acordo com KIRKWOOD e com WHITE [9, 10, 14]

\begin{tabular}{ccc}
\hline $\mathrm{N}^{\mathrm{O}}$ da \\
amostra & $\begin{array}{c}\text { Classificação conforme } \\
\text { Kirkwood }\end{array}$ & $\begin{array}{c}\text { Classificação conforme } \\
\text { White }\end{array}$ \\
\hline 1 & mel floral & mel floral \\
2 & mel floral & mel floral \\
3 & mel floral & mel floral \\
4 & mel de melato & mel de melato \\
5 & mel de melato & mel de melato \\
6 & mel de melato & mel de melato \\
7 & mel de melato & mel de melato \\
8 & mel de melato & mel de melato \\
9 & mel floral & mel floral \\
10 & mel de melato & mel floral \\
11 & mel floral & mel de melato \\
12 & mel de melato & mel floral \\
13 & mel de melato & mel de melato \\
14 & mel floral & mel floral \\
15 & mel de melato & mel de melato \\
16 & mel floral & mel floral \\
17 & mel floral & mel floral \\
18 & mel floral & mel floral \\
19 & mel floral & mel floral \\
20 & mel floral & mel floral \\
21 & mel floral & mel floral \\
22 & mel de melato & mel de melato \\
23 & mel de melato & mel de melato \\
24 & mel floral & mel de melato \\
25 & mel floral & mel floral \\
& & \\
& &
\end{tabular}

Das 11 amostras classificadas como mel de melato de acordo com Kirkwood, duas (no 10 e 12) eram mel floral, de acordo com White. Da mesma forma, das 11 amostras classificadas como mel de melato, de acordo com White, duas (no 11 e 24) eram mel floral, conforme Kirkwood. Portanto, foi utilizado o teste exato de Fisher, a fim de verificar se há associação entre os dois métodos. A tabela de contingência, a seguir, com as freqüências de mel floral e de melato, de acordo com as duas classificações, levou a um resultado muito significativo, mostrando que há associação entre os dois métodos de classificação. Isto significa que estes critérios de classificação são equivalentes e ambos podem ser usados. Ou seja, o grau de acerto na classificação por qualquer um dos dois testes é de 21 (12+9) em 25 (84\%).

Tabela de contingência para o estudo de associação do tipo de mel e do método de classificação empregado.

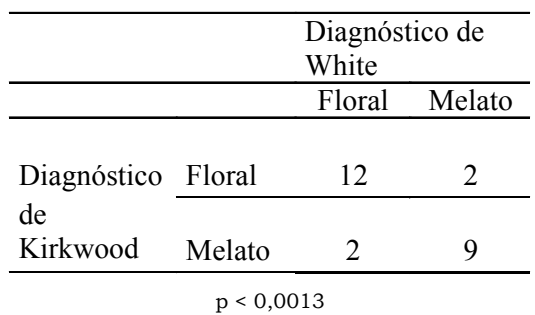

\section{4 - CONCLUSÕES}

Os métodos de Kirkwood e White mostraram-se eficientes para estabelecer se o mel é floral ou de melato.

Os métodos de Kirkwood e de White para classificação de mel em floral ou de melato são equivalentes, de acordo com o teste exato de Fisher.

\section{5 - REFERÊNCIAS}

[1] ABNT - Associação Brasileira de Normas Técnicas. NBR 13922 - Aguardente de cana - Determinação de Açúcares. Agosto de 1997.

[2] AOAC ASSOCIATION OF OFFICIAL ANALYTICAL CHEMISTS. (1980, 1984, 1990, 1995).Official methods of analysis of the Association of Official Analytical Chemists. $13^{\text {th Ed., }}$ Washington D.C.: 1980 - 1995. 1018 p.

[3] BARTH, O.M. O Pólen Brasileiro. Rio de Janeiro: Ed. Luxor, 1989 150p.

[4] BRASIL. Ministério da Agricultura e do Abastecimento. Lanara - Método Analítico Oficial para controle de Produtos de Origem Animal e seus Ingredientes. II Métodos Físicos e Químicos, Brasília, set. 1981.

[5] BRASIL. Ministério da Agricultura. Secretária de Defesa Agropecuária. Instrução Normativa no 11, de 20 de Outubro de 2000. Aprova o Regulamento Técnico de Identidade e Qualidade do mel. Publicado no DOU de 23 de Outubro de 2000, Seção I, p. 16-17.

[6] CAMPOS, G. Melato no mel e sua determinação através de diferentes metodologias: (Tese de Doutorado em Ciência Animal), Escola de Veterinária - UFMG, Belo Horizonte 1998, 178 p. 
[7] DONER, W. L. The sugars of Honey - A review J. Sci. Food. Agric .v. 28, p. 443 - 456, 1977.

[8] PUTTKAMMER, E.: Presidente da Federação das Associações de Apicultores de Santa Catarina. Informação Pessoal. 2000.

[9] KIRKWOOD, K. C., MITCHELL, T. J., SMITH, D. Examination of the occurrence of honeydew in honey. Analyst, v. 85, p. 412-416, 1960.

[10] KIRKWOOD, K. C., MITCHELL, T. J., SMITH, D. Examination of the occurrence of honeydew in honey. Part II Analyst, v. 85 , p. $164-165,1961$.

[11] NORMAS ANALÍTICAS DO INSTITUTO ADOLFO LUTZ. Métodos químicos e físicos para análise de alimentos. $3^{\text {a }}$ ed. São Paulo, Instituto Adolfo Lutz, 1985. v.1.

[12] SALOMÉ, L. G. EPAGRI, Cidade das abelhas, S.C. Brasil. Informação pessoal, setembro, 1996.

[13] SIDDIQUI, I. R. The sugars of honey. Adv. Carbohydr. Chem. n. 25, p. 285 - 288, 1970.

[14] WHITE Jr., J.W. Detection of Honey Adulteration By Carbohydrate Analysis. J. Assoc. Off. Anal. Chem., v. 63, n.1, 1980.

\section{6 - AGRADECIMENTOS}

À FAPEMIG pelo apoio financeiro. 